\title{
Long-lived phonon polaritons in hyperbolic materials
}

G. X. Ni ${ }^{1,2, \dagger}$, A. S. McLeod ${ }^{3}$, Z. Sun ${ }^{3}$, J. Matson ${ }^{4}$, L. Lo $^{2}$, D. A. Rhodes ${ }^{5}$, F. L. Ruta ${ }^{3,6}$, R. A. Vitalone $^{3}$, L. Xiong 3 , S. L. Moore ${ }^{3}$, R. Cusco ${ }^{7}$, L. Artús ${ }^{7}$, C. R. Dean ${ }^{3}$, J. Hone ${ }^{5}$, A. J. Millis ${ }^{3}$, M. M. Fogler ${ }^{8}$, J. H. Edgar ${ }^{9}$, J. D. Caldwell ${ }^{4}$, D. N. Basov ${ }^{3, \dagger}$

${ }^{1}$ Department of Physics, Florida State University, Tallahassee, Florida 32306, USA.

${ }^{2}$ National High Magnetic Field Laboratory, Tallahassee, Florida 32310, USA.

${ }^{3}$ Department of Physics, Columbia University, New York, NY 10027, USA.

${ }^{4}$ Department of Mechanical Engineering, Vanderbilt University, Nashville, Tennessee 37235, USA.

${ }^{5}$ Department of Mechanical Engineering, Columbia University, New York, NY 10027, USA.

${ }^{6}$ Department of Applied Physics and Applied Mathematics, Columbia University, New York, NY 10027, USA.

${ }^{7}$ GEO3BCN-CSIC, Lluís Solé i Sabarís s.n., 08028 Barcelona, Spain.

${ }^{8}$ Department of Physics, University of California, San Diego, La Jolla, California 92093, USA.

${ }^{9}$ Tim Taylor Department of Chemical Engineering, Kansas State University, Manhattan, KS 66506, USA.

†Corresponding author: guangxin.ni@magnet.fsu.edu,db3056@columbia.edu.

Natural hyperbolic materials with dielectric permittivities of opposite sign along different principal axes can confine long-wavelength electromagnetic waves down to the nanoscale, well below the diffraction limit. This has been demonstrated using hyperbolic phonon polaritons (HPP) in hexagonal boron nitride (hBN) and $\alpha-\mathrm{MoO}_{3}$, among other materials. However, HPP dissipation at ambient conditions is substantial and its fundamental limits remain unexplored ${ }^{1,2}$. Here, we exploit cryogenic nano-infrared imaging to investigate propagating HPP in isotopically pure hBN and naturally abundant $\alpha-\mathrm{MoO}_{3}$ crystals. Close to liquid-nitrogen temperatures, the losses for HPP in isotopic hBN drop significantly, resulting in propagation lengths in excess of 25 micrometers, with lifetimes exceeding 5 picoseconds, thereby surpassing prior reports on such highly-confined polaritonic modes. Our nanoscale, temperature-dependent imaging reveals the relevance of acoustic phonons in hyperbolic polariton damping and will be instrumental in mitigating such losses for miniaturized middle infrared technologies operating at the liquid-nitrogen temperatures. 
We investigate hyperbolic phonon polariton (HPP) propagation and dissipation in isotopically pure hexagonal boron nitride $\left(\mathrm{h}^{10} \mathrm{BN}, \mathrm{h}^{11} \mathrm{BN}\right)$, along with naturally abundant $\mathrm{hBN}$ and in $\alpha-\mathrm{MoO}_{3}$ van der Waals crystals using near-field infrared (IR) microscopy. Nano-IR methods allow one to directly visualize polaritonic standing waves on the surface of these hyperbolic materials ${ }^{1-7}$. Similar to graphene plasmon polariton investigations ${ }^{8-13}$, both the wavelength of the HPP $\lambda_{p}(\omega)$ and its dissipation $\gamma$ can be readily obtained from nano-IR images (Figs.1-2), with these two quantities allowing one to extract the complex dielectric function $\varepsilon(\omega)=\varepsilon^{\prime}+i \varepsilon^{\prime \prime}$ of a phononic medium $^{3-6}$. Earlier attempts have characterized HPP in isotopically pure $\mathrm{hBN}\left(\mathrm{h}^{10} \mathrm{BN}, \mathrm{h}^{11} \mathrm{BN}\right)$ and in $\alpha-\mathrm{MoO}_{3}$ crystals at ambient conditions ${ }^{6,14-19}$. However, the fundamental limits of HPP dissipation and lifetime remain to be determined as this necessarily relies on temperaturedependent nano-imaging of polaritonic waves. In this work, we report cryogenic nano-imaging results for these van der Waals materials for the first time, demonstrating record-long HPP lifetimes. We studied isotopically pure $\mathrm{hBN}$ and biaxial hyperbolic $\alpha-\mathrm{MoO}_{3}$ crystals, and compares these results with naturally abundant hBN crystals. Combined with theoretical models, we examined the physics governing HPP dissipation in isotopically pure hyperbolic crystals.

To perform cryogenic nano-imaging, we utilized a home-built scanning near-field IR microscope operating at variable temperatures ${ }^{8,20-22}$. In this setup, the incident light with IR frequency $\omega$ is focused onto the metallized tip of an atomic force microscope (AFM). As the tip approaches the sample, a concentrated evanescent field excites polaritonic modes with a $\lambda_{p}(\omega)$ that is much shorter than the free-space wavelength $\lambda_{\mathrm{IR}}=2 \pi c / \omega$ of the incident photons $\mathrm{s}^{23-27}$. This unique nano-IR apparatus has been routinely employed in studies of plasmon and phonon polaritons as well as for visualizing inhomogeneities in complex oxides ${ }^{20-22}$. In the previous HPP 
nano-imaging studies, a physical sample edge was chosen as the HPP wave reflector ${ }^{6}$, resulting in the imaging of coexisting fringes with two distinct periodicities $\lambda_{\mathrm{p}}$ and $\lambda_{\mathrm{p}} / 2$. To avoid the complications of $\lambda_{\mathrm{p}}$ and $\lambda_{\mathrm{p}} / 2$ fringe decompositions, an alternative approach is preferred. In our device (Figs.1b), pre-patterned micron-sized Au disk structures residing on top of the hBN crystals (bottom of $\alpha-\mathrm{MoO}_{3}$ ), serve as fixed HPP antennas (Figs. 1,2) predominantly producing fringes with $\lambda_{\mathrm{p}}$ periodicity.

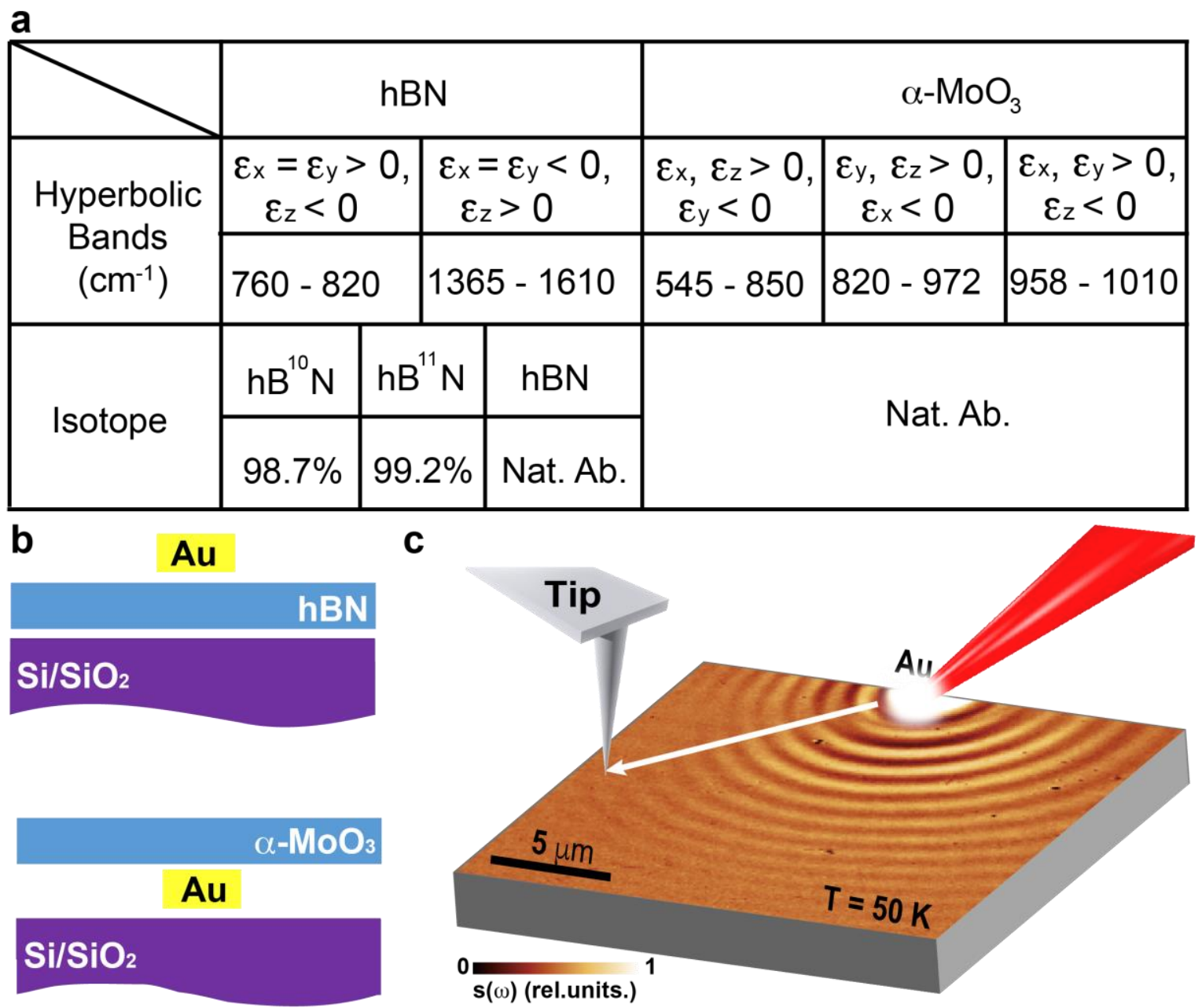

Figure 1. Nano-IR imaging of phonon polaritons in hyperbolic strutures at cryogenic temperatures. a, Table with properties of examined hyperbolic materials $\mathbf{b}$, Sketch of the layered $\mathrm{Au} / \mathrm{hBN}$ and $\alpha-\mathrm{MoO}_{3} / \mathrm{Au}$ structures. The lithographically-defined gold ( $\left.\mathrm{Au}\right)$ microstructures on top of the hBN (or below the $\alpha-\mathrm{MoO}_{3}$ ) serve as effective plasmonic launchers. c, Nanoscale infrared image of phonon polariton fringes from the $\mathrm{Au} / \mathrm{h}^{11} \mathrm{BN}$, expressed by the normalized scattering amplitude $s$ acquired at a temperature of $T=50 \mathrm{~K}$. The arrows represent the propagation 
direction of the plasmon waves. These experiments simultaneously visualize the local electric field associated with interference fringe patterns from phonon polaritons emitted by the Au microstructures.

We begin with a survey of a large-area $\left(25 \times 20 \mu \mathrm{m}^{2}\right)$ image of HPP standing waves in $\mathrm{h}^{11} \mathrm{BN}$ obtained at $T=50 \mathrm{~K}$ (Fig. 1c) with an IR laser operating at $\lambda_{\mathrm{IR}}=6.6 \mu \mathrm{m}$. Here we display raw data in the form of the scattered near-field amplitude $s$ normalized to the corresponding signal detected from the gold disks, whose optical response provides a convenient temperature (T)independent reference. The most prominent aspect of the image in Fig. 1c is that the entire field of view is filled with HPP fringes. As expected, the $\lambda_{\mathrm{p}}$-fringes dominate the field of view, emanating from the Au antenna and propagating radially outwards. Even a cursory inspection of Fig. 1c reveals that HPP remain highly confined with $\lambda_{\mathrm{IR}} / \lambda_{\mathrm{p}}>20$ and yet they travel over tens of microns, far exceeding previous measurements at ambient temperature ${ }^{6,14}$.

Nano-IR data in Fig. 2 attest to a clear reduction of HPP losses in monoisotopic hBN specimens at lower temperatures with the incident frequency of $1522 \mathrm{~cm}^{-1}(6.57 \mu \mathrm{m})$. HPP in $\mathrm{h}^{11} \mathrm{BN}$ films (thickness of $180 \mathrm{~nm}$ ) at room temperature exhibit $15 \mu \mathrm{m}$ propagation lengths, corresponding to quality factor $Q_{\mathrm{p}} \sim 35$ as defined below (Fig 2d\&e). As the temperature is reduced, we observe a systematic increase in quality factor and propagation length. Specifically, at $T=45 \mathrm{~K}, Q_{\mathrm{p}}$ exceeds $\sim 60$ which represents the highest quality factor achieved to date. To elucidate the underlying polariton scattering mechanisms, we have also performed T-dependent studies in $\mathrm{h}^{10} \mathrm{BN}$ and naturally abundant samples for systematic comparisons (Fig. 2a\&c). Two observations can be drawn. First, we observed that the monoisotopic specimens of $\mathrm{h}^{10} \mathrm{BN}$ and $\mathrm{h}^{11} \mathrm{BN}$ share nearly identical propagation lengths at all temperatures. These HPP oscillations exhibit scattering lifetimes 2-3 times longer than those observed in naturally abundant hBN crystals, consistent with prior results ${ }^{6}$. Second, HPP propagation lengths at a given frequency 
generally increase with reduced temperatures for all hBN specimens, as documented in Fig 2d\&e. Notably, we found that both $\mathrm{h}^{10} \mathrm{BN}$ and $\mathrm{h}^{11} \mathrm{BN}$ exhibit a different temperature dependences compared with naturally abundant hBN crystals of similar thickness, as manifested by their HPP oscillations and the quality factor $Q_{\mathrm{p}}(\mathrm{Fig} 2 \mathrm{~d} \& \mathrm{e})$. These overall trends suggest different underlying mechanisms of phonon scattering between isotopically-pure and natural abundant $\mathrm{hBN}$, as discussed in the following sections in detail.
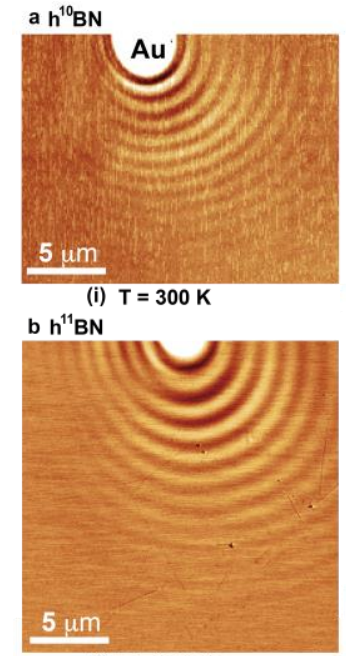

(i) $\mathrm{T}=300 \mathrm{~K}$

c Nat. Ab.

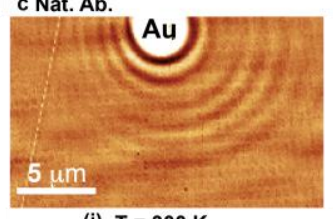

(i) $\mathrm{T}=300 \mathrm{~K}$

d (i) $=h^{10} B N$

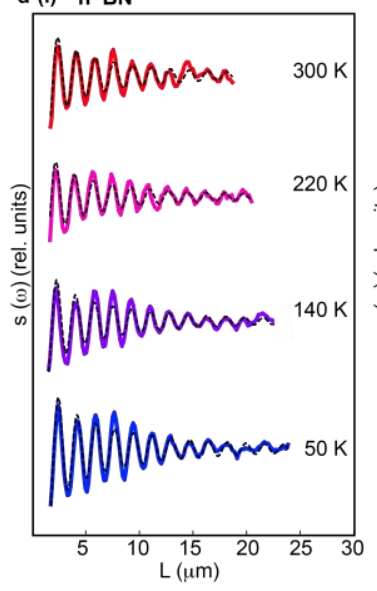

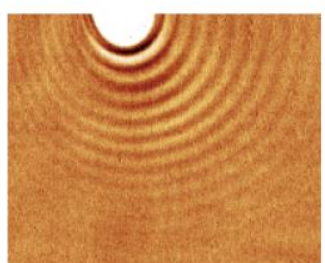

(ii) $\mathrm{T}=220 \mathrm{~K}$

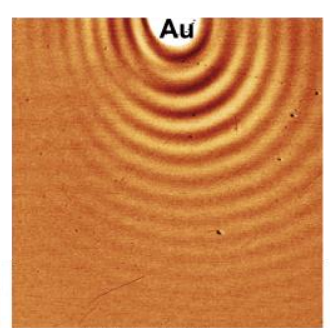

(ii) $\mathrm{T}=220 \mathrm{~K}$

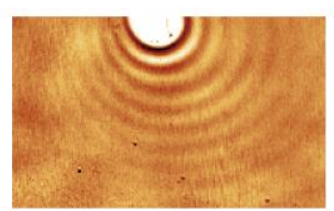

(ii) $\mathrm{T}=220 \mathrm{~K}$

(ii) $=h^{11} \mathrm{BN}$

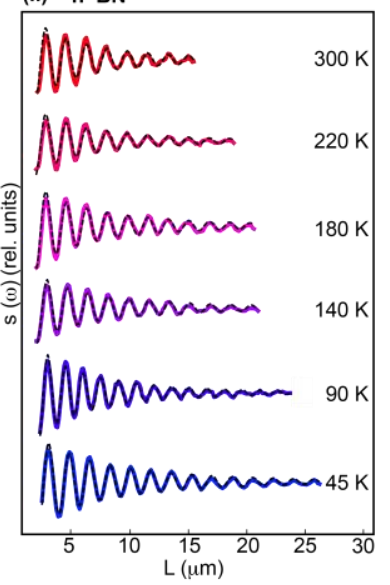

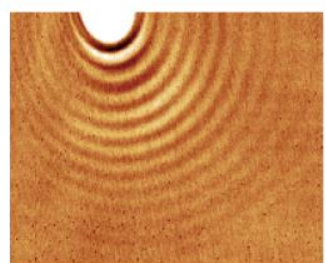

(iii) $\mathrm{T}=140 \mathrm{~K}$

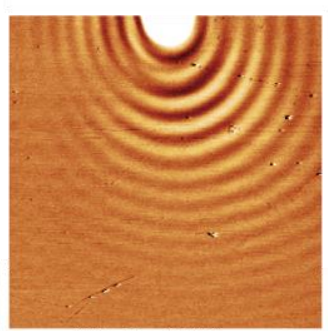

(iii) $\mathrm{T}=140 \mathrm{~K}$

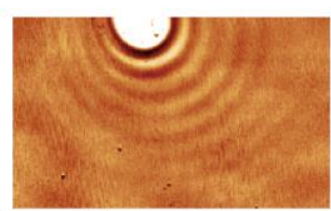

(iii) $T=140 \mathrm{~K}$

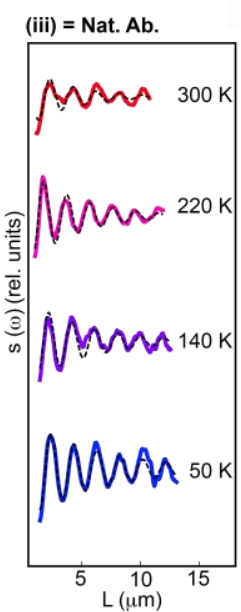

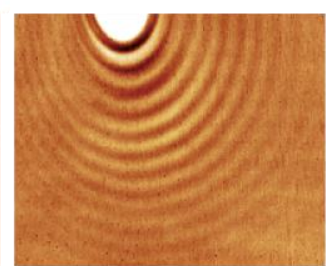

(iv) $\mathrm{T}=50 \mathrm{~K}$

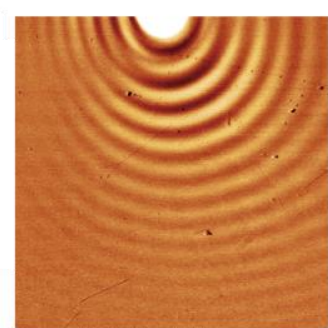

(iv) $\mathrm{T}=45 \mathrm{~K}$

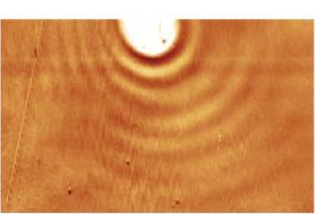

(iv) $\mathrm{T}=50 \mathrm{~K}$

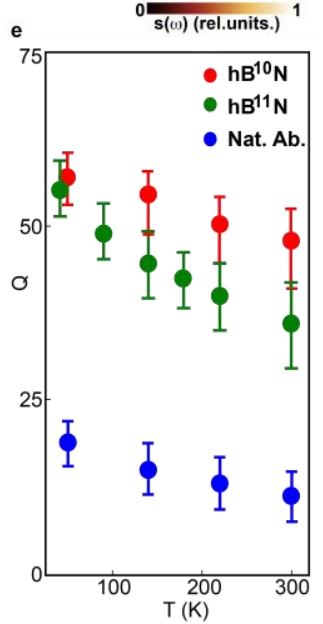


Figure 2. Temperature dependence of phonon polariton propagation in isotopic $h B N$ devices. a-c, Nano-IR images of the normalized scattering amplitude $s$ acquired at sequential sample temperatures from $\mathrm{h}^{10} \mathrm{BN}, \mathrm{h}^{11} \mathrm{BN}$ and natural abundant $\mathrm{hBN}$, respectively. A gold disk (labelled $\mathrm{Au}$ ) functions as an antenna that emits $\mathrm{hBN}$ phonon waves. d, Line profiles of phonon polariton fringes propagating from left to right, as a function of the distance $L$ from the gold launcher. The variable attenuation of the propagating waveform (solid curves) is characteristic of the temperature-dependent phonon damping rate. Dash-dotted lines are fittings of the wave profile using damped sinusoidal functions corrected by geometic decaying factors (see main text and Methods). e, The temperature dependence of the quality factor $Q_{\mathrm{p}}$ for phonon waves obtained from nanoscale infrared images shown in a-d. The thickness of $h^{10} \mathrm{BN}, \mathrm{h}^{11} \mathrm{BN}$ and naturally abundant hBN flakes are $150 \mathrm{~nm}, 200 \mathrm{~nm}$ and $180 \mathrm{~nm}$, respectively.

For contrast, we have also investigated temperature dependence of hyperbolic HPP in exfoliated crystals of $\alpha-\mathrm{MoO}_{3}$, an emerging natural material that exhibits in-plane hyperbolicity at mid-IR frequencies, to determine if the same temperature dependent scattering is observed as in $\mathrm{hBN}^{14,15,17}$. In contrast to the convex wavefronts in isotropic materials, a concave wavefront of the HPP modes in thin slabs of $\alpha-\mathrm{MoO}_{3}$ have been observed in ambient conditions ${ }^{14,15,17,18}$. In Fig. 3, we show raster scans of an $\alpha-\mathrm{MoO}_{3}$ crystals ( $200 \mathrm{~nm}$ thickness) exfoliated onto an $\mathrm{Au}$ antenna obtained at temperatures ranging from 50 to $300 \mathrm{~K}$ and at the frequency of $928 \mathrm{~cm}^{-1}$. At room temperature, HPP interference fringes with concave shapes along the (100) direction were observed, consistent with the isofrequency curves as expected from the in-plane hyperbolic responses ${ }^{14,15,17}$. As the temperature is reduced, we observe a systematic increase in both the overall propagation distance and the number of detectable HPP oscillations, consistent with hBN results. At $T=50 \mathrm{~K}$, HPP oscillations approach propagation lengths of $20 \mu \mathrm{m}$ (Fig. 3a). Similar temperature-dependence of HPP was also detected in the elliptical response regime (see SI). These overall trends are similar to our results on hBN isotopes, indicating the uniform characteristics of phonon propagation and dissipation emerging at low temperatures in hyperbolic crystals. 


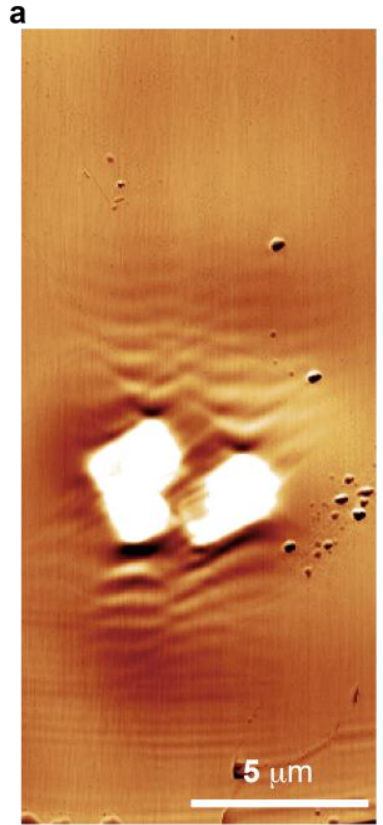

(i) $\mathrm{T}=300 \mathrm{~K}$

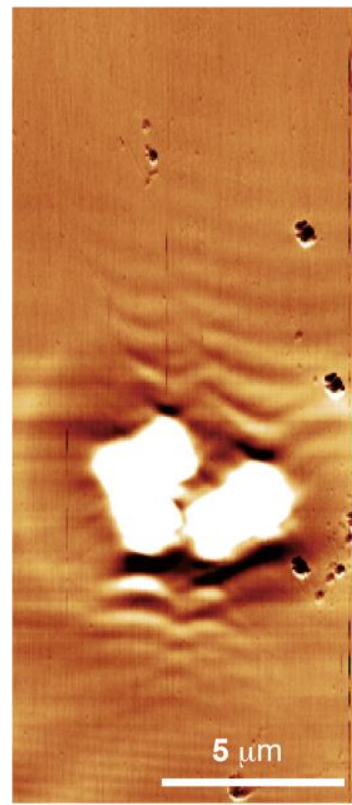

(ii) $\mathrm{T}=220 \mathrm{~K}$

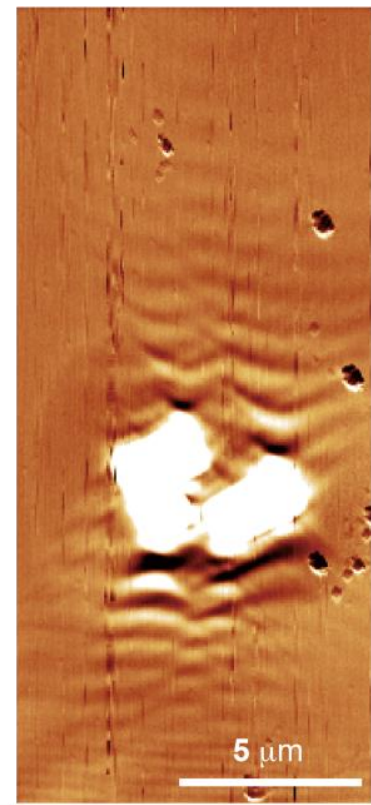

(iii) $\mathrm{T}=140 \mathrm{~K}$

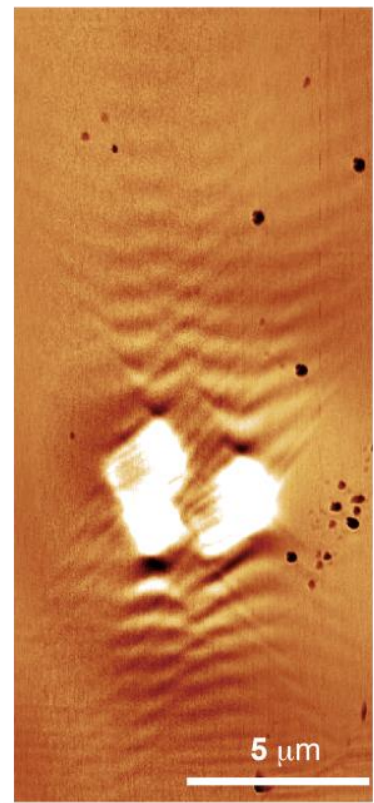

(iv) $\mathrm{T}=50 \mathrm{~K}$

0 s( $\omega)$ (rel.units.)

b

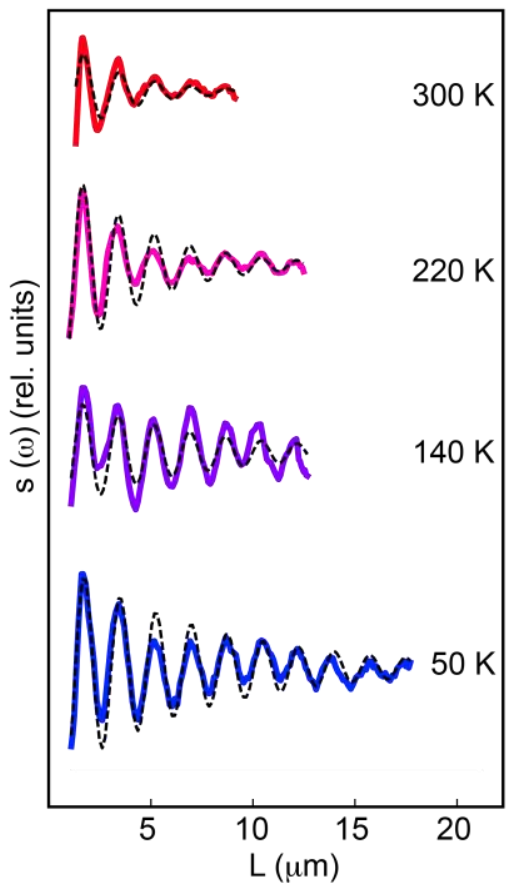

c

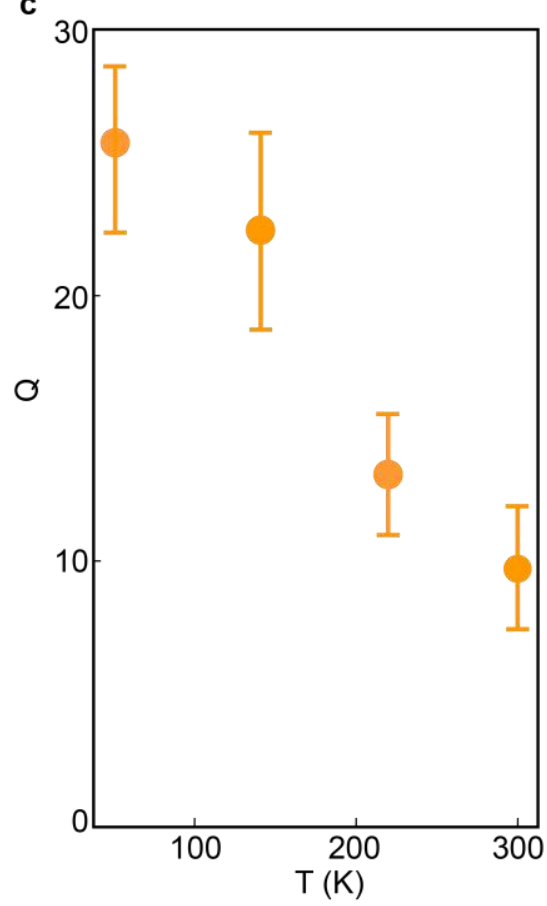

Figure 3. Temperature dependence of phonon polariton propagation in $\alpha$-MoO3 crystals. a, Nano-IR images of the normalized near-field scattering amplitude $s$ acquired at sequential sample temperatures from $\alpha-\mathrm{MoO}_{3}$ crystals. A pair of gold disks beneath the $\alpha-\mathrm{MoO}_{3}$ crystal function as antennas that emit polaritonic waves. $\mathbf{b}$, Line profiles of phonon polariton fringes propagating from left to right, as a function of the distance $L$ from the gold launcher. Dash-dotted lines represent the results of numerical simulations performed to identify the temperature dependence of the complex 
phonon polariton wavevector and associated damping rate (see main text and Methods). c, Temperature dependence of the quality factor $Q_{\mathrm{p}}$ of phonon waves obtained from nanoscale infrared images shown in a.

To quantify the dynamics underlying propagation of HPP in $\mathrm{hBN}$ and $\alpha-\mathrm{MoO}_{3}$ crystals, we present an analysis based on the complex momentum of the polariton $q_{p}=q_{p}^{\prime}+i q_{p}^{\prime \prime}$. The real part defines the wavelength through $\lambda_{p}=2 \pi / q_{p}^{\prime}$, whereas the imaginary part $q_{p}^{\prime \prime}$ quantifies dissipation and the quality factor $Q_{\mathrm{p}}=q_{p}^{\prime} / q_{p}^{\prime \prime}$. To quantify $Q_{\mathrm{p}}$, we fit the oscillating line-profiles away from the $\mathrm{Au}$ antenna employing the spheroidal model with analytical formula of $S(x)=$ $\frac{A \sin \left(q_{p}^{\prime} x+B\right) e^{-q_{p}^{\prime \prime} x}}{\sqrt{x+\zeta}}$, with complex paramters $q_{p}$ and real A, B and $\zeta$ as fitting parameters ${ }^{26}$ (see SI for details). This analytical formula accounts for fringe decay processes away from the antenna with the presence of a finite damping $\left(q_{p}^{\prime \prime}>0\right)$ and a geometric spreading term in the denominator. As shown by solid curves superimposed on polariton line-profiles in Fig. 2 d \& Fig. 3b, the fitted results match well with the experimental data across variable temperatures. This analysis allowed us to extract the temperature-dependent quality factors $Q_{\mathrm{p}}(T)$ for polaritons in all isotopes of hBN and $\alpha-\mathrm{MoO}_{3}$ crystals for the first time, as presented in Fig. 2e \& Fig. 3c. Specifically, we found that the highest quality factor $Q_{\mathrm{p}}(T)$ stems from the near-monoisotopic hBN flakes at cryogenic temperatures with $Q_{\mathrm{p}}$ exceeding 60 .

We now outline the damping rate analysis based on the temperature-dependent results extracted from hBN crystals. In Fig. 4, we plot the temperature-dependent HPP damping rates (linewidth) $\gamma(T)=\omega v_{g} / Q_{p}(T) v_{p}$ converted from quality factors, where $v_{\mathrm{g}}$ and $v_{\mathrm{p}}$ are the group and phase velocities of the modes. Note that the damping rates are on the order of several wavenumbers (approaching $\omega / 1000$ ), while the quality factors defined in terms of momenta are 
relatively small due to the very low group-phase velocity ratio: $\frac{v_{g}}{v_{p}} \sim 0.04$ (obtained from theoretical HPP dispersions, see SI). Several pieces of information arise from Fig. 4. Firstly, the HPP damping rates scale quasi-linearly with temperature for all samples. This is different from the nonlinear temperature dependence of plasmon damping rates observed in pristine graphene ${ }^{8}$. Secondly, compared to $\mathrm{h}^{11} \mathrm{BN}$ and $\mathrm{h}^{10} \mathrm{BN}$, the polariton damping rate in naturally abundant $\mathrm{hBN}$ displays a higher residual damping in the zero-Kelvin limit and a steeper slope to the temperature dependence.

The temperature dependent studies offer insights into the damping mechanisms of HPP in both $\mathrm{hBN}$ and $\alpha-\mathrm{MoO}_{3}$ crystals. We posit that the temperature dependence can only be described by the participation of acoustic phonons in the scattering process, as the optical phonons are not thermally occupied in the temperature range of $T<300 \mathrm{~K}=208 \mathrm{~cm}^{-1}$ (see SI). In isotopically pure $\mathrm{hBN}$, we attribute the leading damping channel to momentum relaxation caused by acoustic phonons, that is, the polariton being scattered from its intial state with momentum $k_{i}$ to the final state with momentum $k_{f}=k_{i}+q$ by absorbing/emiting an acoustic phonon with momentum $\pm q$ (Fig. 4c). Under reasonable approximations (see SI Sec. 4.1), the resulting scattering rate reads $\gamma_{a}(T) \sim \gamma_{a 0}(\omega) f\left(\frac{T}{T_{\Lambda}}\right)$, where $\gamma_{a 0}(\omega)$ is a characteristic scattering rate, $T_{\Lambda}=v_{a} \Lambda / k_{B}$ is a temperature 'cutoff', $v_{a}$ is the speed of acoustic phonons and $\Lambda$ is the momentum cutoff of the hyperbolic polaritons. The temperature dependence is captured by the scaling function $f\left(\frac{T}{T_{\Lambda}}\right)$, which behaves as $1+\frac{8 \pi^{4}}{15}\left(\frac{T}{T_{\Lambda}}\right)^{4}$ when $T \ll T_{\Lambda}$ and as $1+\frac{8}{3} \frac{T}{T_{\Lambda}}$ when $T \gg T_{\Lambda}$.

Isotopic impurities in natural $\mathrm{hBN}$ can also induce the same decay process as acoustic phonons, giving rise to a temperature-independent scattering rate $\gamma_{i}(\omega)$. Both $\gamma_{i}(\omega)$ and 
$\gamma_{a 0}(\omega)$ are proportional to the polariton density of states $D(\omega)=\partial_{\omega} \sqrt{-\frac{\epsilon_{x}}{\epsilon_{z}}}$, where $\epsilon_{x, z}$ are the inplane/out-of-plane dielectric functions of $\mathrm{hBN}$. Here $D(\omega)$ diverges as $\frac{1}{\left(\omega-\omega_{T O}\right)^{3 / 2}}$ when the frequency approaches the transverse optical (TO) phonon, while weakly depending on $\frac{1}{\left(\omega_{L O}-\omega\right)^{1 / 2}}$ as the frequency approaches the longitudinal optical (LO) phonons. Such a strong frequency dependence suggests that the frequency-dependent damping rate $\gamma(\omega)$ needs to replace its constant counterpart in the Lorentzian oscillator model. Note that polaritons with frequency $\omega$ can also decay into $E_{1 u}$ TO phonons or $E_{2 g}$ phonons (nearly flat dispersion at the frequency $\omega_{T O}=\omega_{g}=$ $1360 \mathrm{~cm}^{-1}$ ) by emitting an acoustic phonon. This contributes to a scattering rate $\gamma_{g}(\omega, T)=$ $\gamma_{g 0}(\omega)\left(n_{b}\left(\omega-\omega_{T O}\right)+1\right)$ where the boson occupation number $n_{b}$ gives the temperature dependence and $\gamma_{g 0}$ is a frequency scale depending on $\omega$. However, our theoretical modeling suggests that this term is negligibly small (see SI, section 4). The functional forms of $\gamma_{a}, \gamma_{i}$ and $\gamma_{g}$ therefore represent these new results.

The steeper temperature-dependence of damping rate in natural hBN implies a higher-order decay channel involving both impurities and acoustic phonons, which we subsume by $\gamma_{\mathrm{h}}(T)=$ $\kappa_{\mathrm{i}}(\omega)\left(\gamma_{a}(\omega, T)+\gamma_{g}(\omega, T)\right)$ (see SI). The total polariton damping rates for natural and isotopically pure $\mathrm{hBN}$ are thus

$$
\gamma_{i s o}(T)=\gamma_{0}+\gamma_{a}(T)+\gamma_{g}(T), \text { and } \gamma_{n a t}(T)=\gamma_{i s o}(T)+\gamma_{i}+\gamma_{\mathrm{h}}(T)
$$

where $\gamma_{0}$ is a temperature-independent scattering rate due to other decay processes ${ }^{28,29}$. The dashed fitting lines in Figure 4 correspond to $\gamma_{0}=0.4 \mathrm{~cm}^{-1}, \gamma_{a 0}=0.6 \mathrm{~cm}^{-1}, \gamma_{g 0}=0, \gamma_{i}=$ $0.5 \mathrm{~cm}^{-1}, \kappa_{\mathrm{i}}=2.5$ and $T_{\Lambda}=450 \mathrm{~K}$. Note that these fitting parameters are obtained for our simplified analytical model. Further dedicated first-principle calculations are possibly needed for 
fine tuning, which is beyond the scope of the current work. On top of the above intrinsic damping channels, the dielectric loss from the $\mathrm{SiO}_{2}$ substrate contributes $<20 \%$ to the total damping rate at the experimental frequency of $1522 \mathrm{~cm}^{-1}$. Finally, the damping rate in $\alpha-\mathrm{MoO}_{3}$ is smaller than natural hBN, while slightly larger than monoisotopic hBN.
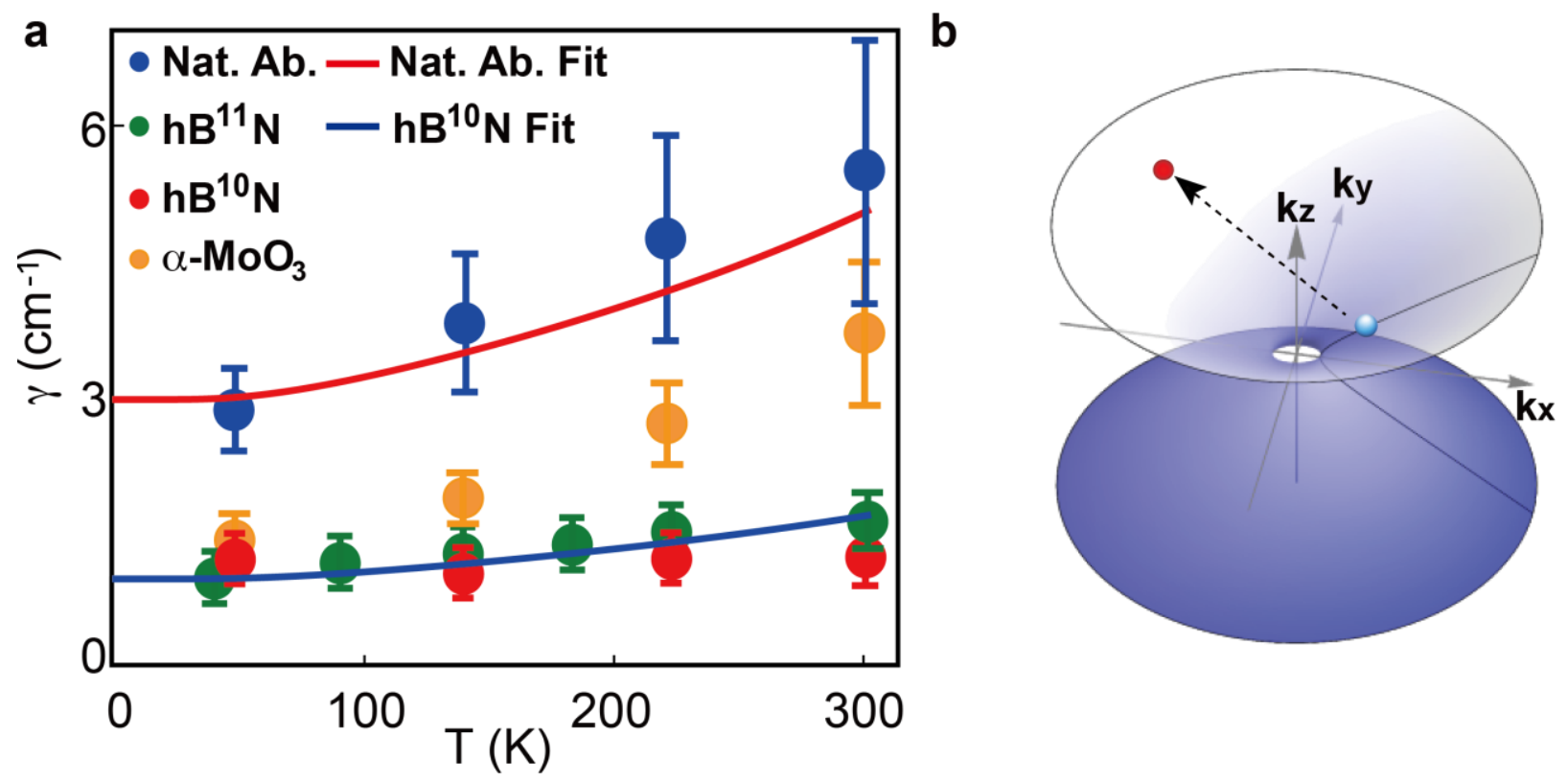

Figure 4. Theoretical modeling of phonon polariton damping rate $\boldsymbol{\gamma}$ in hyperbolic crystals. (a) The dots corresponding to the extracted dissipation factors as a function of temperature; the solid blue \& red lines are the theoretical fitting results as detailed in the main text. The parameters used here are: $\mathrm{hB}^{11} \mathrm{~N}: \omega=1522 \mathrm{~cm}^{-1}, v_{\mathrm{g}} / v_{\mathrm{p}}=0.037 ; \mathrm{hB}^{10} \mathrm{~N}: \omega=1553 \mathrm{~cm}^{-1}, v_{\mathrm{g}} / v_{\mathrm{p}}=0.039$; naturally abundant hBN: $\omega=1522 \mathrm{~cm}^{-1}, v_{\mathrm{g}} / v_{\mathrm{p}}=0.039 ; \mathrm{B}^{11}: \alpha-\mathrm{MoO}_{3}=928 \mathrm{~cm}^{-1}, v_{\mathrm{g}} / v_{\mathrm{p}}=0.039$. The group and phase velocity in $\alpha-\mathrm{MoO}_{3}$ are taken from ref. [13] (b) Key contributions to phonon polariton scattering and their temperature dependences. (c) The illustration of scattering of a hyperbolic polariton from an initial state (blue dot) to a final state (red dot) on the iso-frequency surface by either emitting/obsorbing an acoustic phonon or by impurity potential.

In summary, our results account for HPP dynamics in high-quality hyperbolic crystals. Quality factors and scattering rates of hyperbolic polaritons in naturally abundant and monoisotopic $\mathrm{hBN}$, as well as $\alpha-\mathrm{MoO}_{3}$ have been extracted from cryogenic nano-imaging studies. Though limited by acoustic phonon scattering and other decay channels, the HPP propagation length and lifetime in monoisotopic $\mathrm{hBN}$ at low-T exceed $8 \mu \mathrm{m}$ and $5 \mathrm{ps}$, a new record among all 
natural hyperbolic materials reported so far. Our first report of HPP nano-imaging studies of highquality hyperbolic crystals at cryogenic temperatures demonstrates promising opportunities for the exploration of advanced phononic switching ${ }^{30}$, polariton periodic orbits in cavities ${ }^{31}$ and nonlinear phenomena $^{32}$ in ultra-pure polar samples at technologically relevant liquid nitrogen temperatures. Long-lived polaritons are of interest for applications in infrared signal processing, nanophotonic circuits, nanolight sensing and communications, as well as heat management at the nanoscale ${ }^{14,18}$.

\section{References}

1. Basov, D. N., Fogler, M. M., Garcia de Abajo, F. J., Polaritons in van der Waals materials. Science. 354, aag1992 (2016).

2. Low, T., et al. Polaritons in layered two-dimensional materials. Nature Matter. 16, 182-194 (2017).

3. Caldwell, J. D. et al. Low-loss, infrared and terahertz nanophotonics using surface phonon polaritons. Nanophotonics. 5, 5221 (2014).

4. Dai, S. et al. Tunable phonon polaritons in atomically thin van der Waals crystals of boron nitride. Science 343, 1125-1129 (2014).

5. Li, P. Hyperbolic phonon-polaritons in boron nitride for near-field optical imaging and focusing. Nature Commun. 6, 1-9 (2015).

6. Giles, A. J. et al. Ultralow-loss polaritons in isotopically pure boron nitride. Nature Mater. 17, 134-139 (2018).

7. Basov, D. N. et al. Polaritons Panorama, Nanophotonics 10, 549 (2020).

8. Ni, G. X. et al. Fundamental limits to graphene plasmonics. Nature 557, 530-533 (2018).

9. Jiang, B. Y., Plasmon reflections by topological electronic boundaries in bilayer graphene. Nano Lett. 17, 7080-7085 (2017).

10. Ni, G. X. et al. Plasmons in graphene moiré superlattices. Nature Mater. 14, 1217-1222 (2015).

11. Goldflam, M.D. Tuning and persistent switching of graphene plasmons on a ferroelectric substrate. Nano Lett. 15, 4859-4864 (2015).

12. Sunku, S. S. et al. Photonic crystals for nano-light in moire graphene superlattices. Science 362, 1153-1156 (2018).

13. Fei, Z. Nanoplasmonics phenomena at electronic boundaries in graphene. ACS Photon. 4, 2971-2977 (2017).

14. Ma, W. et al, In-plane anisotropic and ultra-low-loss polaritons in a natural van der Waals crystal. Nature 562, 557-562 (2018).

15. Zheng, Z. et al. A mid-infrared biaxial hyperbolic van der Waals crystal. Science Adv. 5 eaav8690 (2019).

16. $\mathrm{Hu}, \mathrm{G}$. et al. Topological polaritons and photonic magic angles in twisted $\alpha-\mathrm{MoO}_{3}$ bilayers. Nature 582, 209-213 (2020) 
17. Zheng, Z. Highly confined and tunable hyperbolic phonon polaritons in van der Waals semiconducting transition metal oxides. Adv. Mater. 30, 1705318 (2018).

18. Chen, M. et al. Configurable phonon polaritons in twisted $\alpha-\mathrm{MoO}_{3}$. Nature Mater. 19, 13071311 (2020).

19. Duan, J. et al. Twisted nano-optics: manipulating light at the nanoscale with twisted phonon polariton slabs. Nano Lett. 20, 5323-5329 (2020).

20. McLeod, A. S., et al. Nanotextured phase coexistence in the correlated insulator $\mathrm{V}_{2} \mathrm{O}_{3}$. Nature Phys. 13, 80-86 (2017).

21. McLeod, A. S., et al. Multi-messenger nanoprobes of hidden magnetism in a strained magnanite. Nature Mater. 19, 397-404 (2020).

22. Post, K. W. et al. Coexisting first- and second-order electronic phase transitions in a correlated oxide. Nature Phys. 14, 1056-1061 (2018).

23. Ni, G. X. et al. Ultrafast optical switching of infrared plasmon polaritons in high-mobility graphene. Nature Photon. 10, 244-247 (2016).

24. Ni, G. X. et al. Soliton superlattices in twisted hexagonal boron nitride. Nature Commun. 10, 1-6 (2019).

25. Berkowitz M. E. et al. Hyperbolic cooper-pair polaritons in planar graphene/cuprate plasmonic cavities. Nano Lett. 21, 308-316 (2021).

26. Tamagnone, M., Ambrosio, A., Chaudhary, K., Jauregui, L. A., Kim, P., Wilson, W. L. and Capasso, F. Ultra-confined mid-infrared resonant phonon polaritons in van der Waals nanostructures. Science Adv. 4, eaat7189 (2018).

27. Fali, A. et al. Refractive index-based control of hyperbolic phonon polariton propagation. Nano Lett. 19, 7725-7734 (2019).

28. A. Segura, R. Cuscó, T. Taniguchi, K. Watanabe, and L. Artús, Phys. Rev. B 101, 235203 (2020).

29. R. Cuscó, B. Gil., G. Cassabois, and L. Artús. Phys. Rev. B 94, 155435 (2016).

30. Stupakiewicz, A., et al. Ultrafast phononic switching of magnetization. Nature Phys. (2021). Nat. Phys. (2021). https://doi.org/10.1038/s41567-020-01124-9.

31. Sun, Z., A. Gutierrez-Rubio, D. N. Basov, M. M. Fogler. Harmiltonian optics of hyperbolic polaritons in nanogranules. Nano Lett. 15, 4455-4460 (2015).

32. M. Gu., M. Rondinelli. Nonlinear phonic control and emergent magnetism in Mott insulating titanates. Phys. Rev. B 98, 024102 (2018).

\section{Acknowledgments:}

Research at Columbia is supported by Vannevar Bush Faculty Fellowship ONR-VB: N00014-191-2630. We thank A. Sternbach, S. Zhang for helpful discussions. Exfoliation and transfer of hBN onto desired substrates and electron beam lithography of gold disks were performed by J.T.M. and supported through the National Science Foundation (DMR1904793). Additional structure fabrication was supported by by the Center on Precision-Assembled Quantum Materials, funded through the US National Science Foundation (NSF) Materials Research Science and Engineering Centers (award no. DMR-2011738). Initial simulations and experimental design from Vanderbilt was provided by J.D.C. in collaboration with Columbia team (D.N.B. and G.N.) and was supported by the Office of Naval Research (N00014-18-1-2107). hBN phonon band structure calculation was performed by R.C. and L.A. supported through Spanish MINECO/FEDER grant (MAT2015- 
71035-R). Cryogenics nano-optics experiments at Columbia were solely supported as part of Programmable Quantum Materials, an Energy Frontier Research Center funded by the U.S. Department of Energy (DOE), Office of Science, Basic Energy Sciences (BES), under award DESC0019443. D.N.B is the Gordon and Betty Moore Foundation's EPiQS Initiative Investigator \#9455.

\section{Author contributions}

All authors were involved in designing the research, performing the research, and writing the paper.

\section{Additional information}

Supplementary information is available in the online version of the paper. Reprints and permission information is available online at www.nature.com/reprints.

\section{Competing financial interests}

No completing financial interests.

\section{Main Figure Legends}

\section{Methods}

\section{Sample growth and Device fabrication}

$\mathrm{hBN}$ crystals with the natural distribution of isotopes (20\% B-10 and $80 \% \mathrm{~B}-11)$ were produced using a hot-pressed boron nitride ceramic boat, which served as both the container for the metal flux and as the $\mathrm{B}$ and $\mathrm{N}$ sources. For $\mathrm{B}^{10}$ and $\mathrm{B}^{11}$-enriched $\mathrm{hBN}$ crystal growth, the procedure is modified to use elemental boron as a source material. Therefore, the boron nitride boat was replaced with an alumina crucible. High-purity 10B $(99.22 \%)$ or 11B $(99.41 \%)$ powders were mixed with $\mathrm{Ni}$ and $\mathrm{Cr}$ powders to give overall concentrations of $4 \% \mathrm{~B}, 48 \% \mathrm{Ni}$ and $48 \% \mathrm{Cr}$. In this case, all nitrogen in the $\mathrm{hBN}$ originated from the flowing $\mathrm{N}_{2}$ gas. Other than these changes, the procedure was the same as described above. The hBN crystals was then transferred onto $\mathrm{SiO}_{2} / \mathrm{Si}$ substrates using the mechanical exfoliation method. After identifying the crystal thickness and unifomaty, standard E-beam lithography is utilized to define Au launchers followed by thermal annealing processes to remove comtaminated polymer residuals.

\section{Cryogenic IR Nano-Imaging Experiments}

IR nano-imaging experiments were performed using a home-built scattering-type scanning near-field optical microscope (s-SNOM) operating with variable sample temperatures from 50 $300 \mathrm{~K}$. All measurements are conducted under ultra-high vacuum conditions at pressured $<10^{-9}$ mbar to prevent sample surface contamination. The cryogenic s-SNOM is equipped with continuous wave mid-IR quantum cascade lasers (daylightsolutions.com) and $\mathrm{CO}_{2}$ lasers (accesslaser.com). The s-SNOM is based on an atomic force microscope (AFM), operated for the 
present experiments in non-contact mode using cantilevered metallic AFM probes with tip apex radius $\sim 25 \mathrm{~nm}$ and tapping frequencies $\sim 270 \mathrm{kHz}$. A pseudo-heterodyne interferometric detection module is implemented in our s-SNOM to extract both scattering amplitude $s$ and phase of the near-field signal. In the current work, we discuss only the former. In order to suppress background contributions to the back-scattered near-field signal, we demodulated the detected signal at the $3^{\text {th }}$ harmonic of the probe tapping frequency. 